\title{
Cheyne-Stokes-Atmung und kardiovaskuläres Risiko
}

\section{Cheyne-Stokes Respiration and Cardiovascular Risk}

Autoren

Institut
H.-W. Duchna, G. Schultze-Werninghaus

Berufsgenossenschaftliches Universitätsklinikum Bergmannsheil GmbH; Medizinische Klinik III, Pneumologie, Allergologie, Schlaf- und Beatmungsmedizin, Bochum eingereicht 15.4.2009

akzeptiert nach Revision 6. 5.2009

\section{Bibliografie}

DOI 10.1055/s-0029-1214787

Pneumologie 2009; 63:

399-403 @ Georg Thieme Verlag KG Stuttgart · New York ISSN 0934-8387

\section{Korrespondenzadresse \\ Prof. Dr. med.}

Hans-Werner Duchna

Berufsgenossenschaftliches

Universitätsklinikum

Bergmannsheil GmbH

Medizinische Klinik III

Pneumologie, Allergologie,

Schlaf- und Beatmungsmedizin

Bürkle-de-la-Camp-Platz 1

44789 Bochum

hans-werner.duchna@rub.de

\section{Zusammenfassung \\ $\nabla$}

Die Cheyne-Stokes-Atmung (CSR) gehört aufgrund ihrer hohen Prävalenz bei Patienten mit fortgeschrittener Herzinsuffizienz und ihrem negativen Vorhersagewert für die Morbidität und Mortalität der betroffenen Patienten aus pneumologisch-internistischer Sicht zu den wichtigsten schlafbezogenen Atmungsstörungen. Sie ist gekennzeichnet durch ein Crescendo-Decrescendo-Muster der Atmung, kombiniert mit zentralen Apnoephasen, bedingt durch eine Lungenstauung und Oszillationen im Atmungs-Regelkreis. Die CSR gilt als Marker des Schweregrades einer Herzinsuffizienz. Die Therapie der CSR erfolgt nach Optimierung der kardiologischen Therapie der Herzinsuffizienz und der ihr zu Grunde liegenden Ursachen idealerweise mit speziellen, nicht-invasiven Beatmungstherapie-Verfahren. Hierdurch wird eine signifikante Reduktion der nächtlichen CSR einhergehend mit einer Reduktion der Sympathikusaktivität und der Tagesmüdigkeit sowie eine Verbesserung der Herzauswurfleistung und der 6-Minuten-Gehstrecke erreicht. Eine aktuell durchgeführte, multizentrische und prospektiv-randomisierte Langzeit-Interventionsstudie, die Serve-HF-Studie, wird Klarheit über den Nutzen einer Therapie der CSR hinsichtlich des Überlebens der betroffenen Patienten bringen. In dieser Übersichtsarbeit werden Pathophysiologie, Epidemiologie und Interventionsmöglichkeiten der Cheyne-Stokes-Atmung fokussiert auf das kardiovaskuläre Risiko der betroffenen Patienten unter Berücksichtigung des aktuellen Forschungsstandes zusammengefasst.

\section{Abstract \\ $\nabla$}

Due to its high prevalence in patients with heart failure and its negative predictive value concerning morbidity and mortality, Cheyne-Stokes respiration (CSR) is a sleep disorders of major interest. CSR correlates with the degree of heart failure and is characterised by a typical crescendo/decrescendo breathing pattern combined with phases of central sleep apnoea, caused by pulmonary oedema and oscillation of ventilatory control. Thus, CSR is a marker of the severity of heart failure. Treatment of CSR first involves optimisation of heart failure therapy by cardiologists and then application of non-invasive means of ventilatory support. Treatment of patients with severe heart failure with non-invasive positive pressure ventilatory support leads to a significant reduction of CSR, sympathetic activity, and daytime sleepiness and improves cardiac output and 6-minute walking distance. At present, a prospective randomised, controlled intervention-study (Serve-HF study) is being conducted in order to show if therapy of CSR can improve patient survival. This review describes the pathophysiology, epidemiology, and therapeutic options of CSR with a special focus on the elevated cardiovascular risk of patients with CSR. 


\section{Einleitung}

Im Hinblick auf die Prävalenz und die gesundheitlichen Konsequenzen kristallisieren sich in der pneumologisch fokussierten Schlafmedizin drei relevante schlafbezogene Atmungsstörungen (SBAS) innerhalb der im Jahre 2005 von der American Academy of Sleep Medicine (AASM) publizierten Neuauflage der Internationalen Klassifikation von Schlafstörungen (ICSD-2) heraus [1]. Hierbei handelt es sich um das obstruktive Schlafapnoe-Syndrom (OSAS), die Cheyne-Stokes-Atmung (CSR) bei der Herzinsuffizienz und das Obesitas-Hypoventilations-Syndrom (OHS) [2]. Die genannten Krankheitsbilder werden in der Kategorie II der ICSD-2, schlafbezogene Störungen der Respiration, aufgeführt [1]. SBAS einhergehend mit einer (partiellen) Obstruktion der oberen Atmungswege, z. B. das OSAS, werden von SBAS ohne Obstruktion der oberen Atemwege abgegrenzt. Hierzu werden unterschiedliche zentrale Schlafapnoe-Syndrome, z.B. die CSR gezählt.

\section{Pathophysiologie der Cheyne-Stokes-Atmung \\ $\nabla$}

Die von Cheyne (1777 - 1836) und Stokes (1804 - 1878) beschriebene CSR ist primär als Folge einer Linksherzinsuffizienz anzusehen, wird jedoch auch bei pulmonaler Hypertonie [3], Hirninfarkten und Nierenversagen beobachtet [1]. Die Ursachen zentraler Atmungsstörungen sind komplex und vielschichtig [4]. Im Wesentlichen wird davon ausgegangen, dass eine verlängerte Kreislaufzeit bei der Herzinsuffizienz in Kombination mit Veränderungen zentraler und peripherer Chemorezeptorenfunktion vorliegt. Bei herzinsuffizienten Patienten kommt es aufgrund der Lungenstauung via Lungendehnungsreflex zunächst zur Hyperventilation und damit zu einem Abatmen von $\mathrm{CO}_{2}$. Bei Abfall des $\mathrm{PaCO}_{2}$ unter die Apnoeschwelle resultiert eine zentrale Apnoephase mit konsekutivem Anstieg des $\mathrm{PaCO}_{2}$ und Hypoxie [5]. Ein Ansteigen des $\mathrm{PaCO}_{2}$ wiederum führt infolge der verlängerten Kreislaufzeit erst zu einer verspäteten Reaktion des Atemzentrums mit kompensatorischer Hyperventilation [6]. Die verlängerte LungenChemorezeptor-Kreislaufzeit ist direkt proportional zur Länge des Apnoe-Hyperventilations-Zyklus (im Allgemeinen $>45$ Sekunden) und erklärt den graduellen Auf- und Abbau des Atemantriebs [7]. Koprävalente obstruktive Apnoen können während der Nacht aufgrund der repetitiven Sauerstoffdesaturationen, der Sympathikotonuserhöhung und der intrathorakalen Druckschwankungen zu einer zusätzlichen Verschlechterung der kardialen Funktion führen und somit die Entstehung der CSR begünstigen $[4,8-10]$.

Im Gegensatz zur primären zentralen Schlafapnoe, bei der abrupte Apnoe- und kurze Hyperpnoephasen auftreten, weisen Patienten mit zentraler Schlafapnoe mit Cheyne-Stokes-Atmungsmuster ein charakteristisches Crescendo-Decrescendo-Muster ihrer Atmung mit verlängerten Hyperpnoephasen auf. Typischerweise tritt die CSR im non-REM(rapid-eye-movement)-Schlaf auf und kann zu exzessiver Tagesmüdigkeit, insomnischen Beschwerden oder nächtlichen Atemnotbeschwerden führen ([11], ๑ Tab. 1).

Als Risikofaktoren für das Auftreten einer CSR bei Herzinsuffizienz gelten das männliche Geschlecht, ein Alter > 60 Jahre, Vorhofflimmern und Hypokapnie (Tages- $\mathrm{PaCO}_{2} \leq 38 \mathrm{~mm} \mathrm{Hg}$ ) [12]. In einer aktuellen Untersuchung an einem kleinen Kollektiv wird eine Korrelation der CSR zur Diffusionskapazität und dem Hypoxämiegrad am Tage beschrieben [13]. Die CSR gilt als Marker des Schweregrades einer Herzinsuffizienz und ist ein negativer
Tab. 1 Definition der Cheyne-Stokes-Atmung gemäß ICSD-2 [1].

Zentrale Schlafapnoe mit Cheyne-Stokes-Atmung

(Diagnosekriterien nach [1])

Polysomnografie:

$\geq 10$ zentrale Apnoen/h mit Crescendo-Decrescendo-Muster der Atmung assoziiert mit gehäuften Weckreaktionen und gestörter Schlafstruktur Fakultativ: exzessive Tagesmüdigkeit, insomnische Beschwerden, nächtliches Erwachen mit Atemnot

Assoziation mit schwerer internistischer/neurologischer Erkrankung (Herzinsuffizienz, Niereninsuffizienz, Apoplex)

Erkrankung nicht besser beschrieben durch andere Schlafstörung oder

Medikamenten-/Drogengebrauch

\section{CSR: Mortalität $\uparrow$ bei chron. Herzinsuffizienz}

Sympathikotonus $\uparrow$

Repetitive Hypoxämie/Reoxigenierung

Vorhofflimmern

Ventrikuläre Ektopie

Ventrikuläre Tachykardie
Abb. 1 Faktoren, die die Mortalität bei herzinsuffizienten Patienten mit CSR erhöhen [14].
Prognoseparameter, da die CSR bei herzinsuffizienten Patienten zu einer Aktivierung des Sympathikotonus führt sowie das Auftreten von Vorhofflimmern und ventrikulären Ektopien begünstigt $($ Abb. 1) $[2,14,15]$.

Inwieweit die CSR eine proatherogene Stoffwechsellage bedingt, durch repetitive Sauerstoffentsättigungen mit konsekutiver Reoxigenierung einhergehend mit einer Aktivierung proinflammatorischer Marker sowie der Generierung von Sauerstoffradikalen ist, anders als beim OSAS, aktuell nicht eindeutig belegt $[2,16]$.

\section{Epidemiologie der Cheyne-Stokes-Atmung \\ $\nabla$}

In Deutschland war im Jahr 2006 eine Herzinsuffizienz mit 317000 Fällen der häufigste Aufnahmegrund für eine stationäre Behandlung [17]. Eine Herzinsuffizienz war nach Angaben des Statistischen Bundesamtes im selben Jahr die dritthäufigste Todesursache in Deutschland. Die 5-Jahres-Mortalität der chronischen Herzinsuffizienz liegt zwischen 40 und 60 Prozent [18]. In diesem Zusammenhang rücken SBAS bei Patienten mit einer Herzinsuffizienz zunehmend in das medizinische Interesse. Zwei aktuelle, in Deutschland durchgeführte Studien zeigten eine hohe Prävalenz (> 70 Prozent) von SBAS bei Patienten mit stabiler, nach aktuellen Therapierichtlinien medikamentös behandelter, schwerer chronischer Herzinsuffizienz, wobei obstruktive und zentrale Apnoen (CSR) mit annähernd gleicher Häufigkeit beobachtet wurden $[19,20]$.

In die eine Studie wurden 700 Patienten mit einer symptomatischen Herzinsuffizienz (NYHA-Klasse $\geq$ II, linksventrikuläre Ejektionsfraktion $[\mathrm{LV}-\mathrm{EF}] \leq 40$ Prozent) des Herz- und Diabeteszentrums Nordrhein-Westfalen, Universitätsklinik der Ruhr-Universität Bochum, eingeschlossen. In der prospektiven Untersuchung wurde eine nächtliche kardiorespiratorische Polygrafie (nicht-laborgebundenes Monitoring des Schlafs), eine Echokardiografie, eine Spiroergometrie und ein 6-Minuten-Gehtest durchgeführt. SBAS wurden bei 76 Prozent (40 Prozent zentrale Apnoen/CSR; 36 Prozent obstruktive Apnoen [OSA]) der Patienten gesehen. Patienten mit CSR waren dabei einer höheren NYHA-Klasse zugehörig und wiesen eine signifikant niedrigere LV-EF und eine geringere kardiopulmonale Leistungsfähigkeit (Sauerstoffaufnah- 
me, 6-Minuten-Gehstrecke) als Patienten mit überwiegend obstruktiven Apnoen bzw. ohne SBAS auf [19]. Die zweite aktuelle Studie zur Prävalenz von SBAS bei Patienten mit chronischer Herzinsuffizienz wurde multizentrisch innerhalb der Arbeitsgruppe Kreislauf und Schlaf der Deutschen Gesellschaft für Schlafforschung und Schlafmedizin (DGSM) an 10 Standorten in Deutschland durchgeführt. Eingeschlossen wurden 203 Patienten mit einer chronischen Herzinsuffizienz der jeweils stabilen, medikamentös nach aktuellen Therapierichtlinien behandelten NYHA-Stadien II und III (LV-EF < 40\%). Durchgeführt wurde eine nächtliche Polygrafie, deren Auswertung zentral, verblindet ohne Kenntnis der Patientencharakteristika, visuell erfolgte. Von den durchschnittlich 65 Jahre alten Patienten wiesen 71 Prozent $(\mathrm{n}=145)$ schlafbezogene Atmungsstörungen auf, definiert als Apnoe-Hypopnoe-Index (AHI) $>10$ pro Stunde. OSA wurden bei 43 Prozent $(n=88)$, CSR bei 28 Prozent $(n=57)$ der Studienteilnehmer festgestellt. Patienten mit OSA waren signifikant übergewichtig und litten durchschnittlich mehr unter den Symptomen der Tagesmüdigkeit, wohingegen Patienten mit CSR signifikant häufiger ein Vorhofflimmern und einen signifikant niedrigeren $\mathrm{PaCO}_{2}$-Wert am Tage aufwiesen [20]. CSR wird jedoch nicht nur während des Schlafes beobachtet, sondern kann auch während der Wachphase am Tage oder bei körperlicher Anstrengung (periodische Atmung) bei Patienten mit Herzinsuffizienz auftreten. CSR am Tage oder bei körperlicher Belastung korreliert mit dem Schweregrad der Herzinsuffizienz und bedeutet eine signifikant schlechtere Überlebenswahrscheinlichkeit im Vergleich zu isoliert nächtlich auftretender CSR [21,22]. So weisen herzinsuffiziente Patienten mit CSR eine signifikant höhere Mortalität als herzinsuffiziente Patienten ohne CSR auf [23,24].

\section{Therapie der Cheyne-Stokes-Atmung}

$\nabla$

Vor dem Hintergrund einer epidemiologisch relevanten Prävalenz sowohl einer Herzinsuffizienz als auch der SBAS sowie insbesondere der CSR bei herzinsuffizienten Patienten und deren negativem prognostischen Vorhersagewert für das Überleben der Patienten ist die Etablierung von Therapierichtlinien erforderlich. Dabei kann eine abschließende Bewertung des Stellenwertes der Therapie von CSR aufgrund der noch ausstehenden Studien derzeit noch nicht erfolgen. Konsens herrscht jedoch weitgehend über das therapeutische Prozedere bei Patienten mit Herzinsuffizienz und CSR. Zunächst wird die maximale Ausschöpfung kardiologisch-internistischer und ggf. operativer therapeutischer Maßnahmen zur Optimierung der Herzinsuffizienz empfohlen. Hierzu gehören die medikamentöse Therapie der Herzinsuffizienz, die Revaskularisation, die Herzschrittmacherbzw. kardiale Resynchronisationstherapie, herzklappenrekonstruktive Maßnahmen, die Anwendung sogenannter Assist-Devices (herzunterstützende Pumpverfahren) [25-27]. Führen diese Maßnahmen bereits zum Verschwinden der CSR, so sind lediglich Verlaufskontrollen empfohlen. Sollte die CSR trotz Optimierung der Herzinsuffizienztherapie persistieren, so finden neben einer Sauerstofftherapie unterschiedliche atmungsunterstützende Therapieverfahren (Überdrucktherapie) der CSR Anwendung: CPAP (Continuous Positive Airway Pressure/Positivdruck-(Be)Atmung), BiPAP ${ }^{\circledR}$ (Bilevel Positive Airway Pressure) oder spezielle, auf die CSR abgestimmte Therapieverfahren wie zum Beispiel die adaptive Servoventilation oder die dynamische Bilevel-Therapie [25]. Dabei unterdrücken die speziellen, auf die Therapie der CSR abgerichteten Therapieverfahren, wie die adaptive Servo-

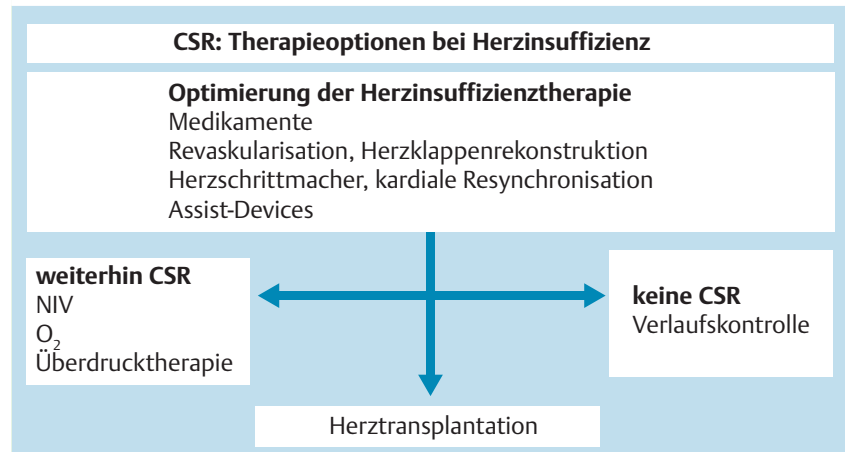

Abb. 2 Algorithmus zur Therapie der CSR bei Patienten mit einer Herzinsuffizienz [25 - 29]. NIV: (nicht-)invasive Beatmung; zu den Verfahren der Überdrucktherapie: siehe Text.

ventilation, die CSR am effektivsten [28]. Bezüglich der intensivmedizinischen Therapie ([nicht-]invasive Beatmung etc.) der akuten respiratorischen Insuffizienz im Rahmen einer akuten Verschlechterung einer Herzinsuffizienz, so zum Beispiel bei der akuten kardialen Dekompensation, sowie der Indikation zu einer Herztransplantation wird auf weiterführende Literatur verwiesen $[26,27,29]$. Die Abb. 2 zeigt einen entsprechenden Algorithmus zur Therapie der CSR bei Patienten mit einer Herzinsuffizienz.

Aus pathophysiologischer Sicht nimmt die kardiale Resynchronisationstherapie, bei der mittels spezieller Herzschrittmacher die kardiale Pumpfunktion durch Optimierung der elektromechanischen Kopplung verbessert wird [30], einen besonderen Stellenwert ein. Langzeitbeobachtungen zeigten positive Effekte einer kardialen Resynchronisationstherapie auf die Reduktion der CSR bei Patienten mit chronischer Herzinsuffizienz. So konnte im Mittel $17 \pm 7$ Wochen nach Implantation eines biventrikulären Herzschrittmachersystems zur kardialen Resynchronisation bei 14 herzinsuffizienten Patienten mit CSR eine signifikante Absenkung des Apnoe-Hypopnoe-Index (AHI) und eine Verbesserung der nächtlichen Sauerstoffsättigung gezeigt werden [31]. Ebenso wurde spiroergometrisch eine Verbesserung der ventilatorischen Effizienz (VE/ $/ \mathrm{VCO}_{2}$-Slope) nach erfolgter kardialer Resynchronisation gezeigt, was im Sinne einer verkürzten Kreislaufzeit zwischen der Lunge und den peripheren Chemorezeptoren sowie einer verbesserten Sensitivität für $\mathrm{CO}_{2} \mathrm{zu}$ interpretieren ist. Weitere Studien bestätigen eine Reduktion der CSR bei chronischer Herzinsuffizienz nach längerfristiger kardialer Resynchronisationstherapie [32,33]. Für die positiven Effekte der kardialen Resynchronisationstherapie auf die CSR und das Überleben der Patienten [34] scheint jedoch nicht die bloße Verbesserung der kardialen Förderleistung ausschlaggebend zu sein, da bei 11 Patienten mit SBAS/CSR unmittelbar nach erfolgter kardialer Resynchronisation keine signifikante Verbesserung hinsichtlich der nächtlichen Atmung bzw. der nächtlichen Atmungsstörung (SBAS/CSR) und der nächtlichen Sauerstoffsättigung nachgewiesen wurde [35]. Erst wenn das Herzschrittmacheraggregat über einen längeren Zeitraum (hier: 2 Monate) implantiert ist, lassen sich Akuteffekte einer kardialen Resynchronisationstherapie durch An- bzw. Ausschalten des Herzschrittmachers nachweisen [36]. Daher scheinen eher chronische Effekte auf die kardiopulmonale Interaktion (Remodelling, Veränderung von Zirkulationszeit und Chemosensitivität) für die Entstehung der CSR bedeutend zu sein.

Die häufige Koprävalenz obstruktiver und zentraler Apnoen bedingt relativ komplexe atmungsunterstützende Therapieverfah- 
ren der SBAS bei herzinsuffizienten Patienten [28], wobei deren Effekte auf die Morbidität und Mortalität von Patienten mit chronischer Herzinsuffizienz noch Gegenstand aktueller Untersuchungen sind. Für Patienten mit CSR bei Herzinsuffizienz konnte in der CANPAP-Studie eine Lebensverlängerung durch eine CPAPTherapie im Gesamtkollektiv nicht gezeigt werden, obwohl die CPAP-Therapie zu einer Verbesserung der nächtlichen Atmung und der Herzleistung führte [37]. Allerdings konnte in einer Subgruppenanalyse der CANPAP-Studie ein Überlebensvorteil derjenigen herzinsuffizienten Patienten gezeigt werden, deren CSR erfolgreich durch eine Überdrucktherapie (unter CPAP: AHI < 15/h) behandelt wurde [38]. Für die speziellen, auf die Therapie der CSR abgerichteten Zweidruck-Beatmungstherapieverfahren ist neben der signifikanten Reduktion der CSR [28,39-41] eine Absenkung des Sympathikotonus und Reduktion der Tagesmüdigkeit [42] sowie eine Verbesserung der Herzauswurfleistung und der 6-Minuten-Gehstrecke $[43,44]$ gezeigt worden. Allerdings sind eine wachsende Anzahl verschiedener Zweidruck-Therapiegeräte mit unterschiedlichen Therapieverfahren auf dem Markt verfügbar. Die unterschiedliche Programmierung der Geräte und Unterschiede in den Therapiealgorithmen sowie die klinische Erfahrung zeigen hier, dass nicht alle Therapieverfahren gleichwertig sind, sodass Studienergebnisse, die mit einem Gerätetyp erzielt wurden, nicht notwendigerweise auf die Therapie mit anderen Therapiegeräten übertragbar sind. So zeigte zum Beispiel die Arbeit von Johnson und Johnson, dass durch eine nicht-adaptive Bilevel-Therapie die Phasen der Hyperventilation sogar verstärkt und damit die CSR verschlechtert werden kann. Daher sind prospektive Studien, die den therapeutischen Nutzen einer CSR-Therapie aufzeigen, unabdingbar. Aktuell wird eine multizentrische und prospektiv-randomisierte Langzeit-Interventionsstudie mit der adaptiven Servoventilation durchgeführt (Serve-HF-Studie). Deren Therapieergebnisse werden möglicherweise die erforderliche Klarheit über den Nutzen einer Therapie der CSR hinsichtlich des Überlebens der Erkrankten bringen [45].

\section{Zusammenfassung \\ $\nabla$}

Die CSR gehört aufgrund ihrer hohen Prävalenz bei Patienten mit fortgeschrittener Herzinsuffizienz und ihrem negativen Vorhersagewert für die Morbidität und Mortalität der betroffenen Patienten aus pneumologisch-internistischer Sicht zu den wichtigsten schlafbezogenen Atmungsstörungen. Die CSR gilt daher als Marker des Schweregrades einer Herzinsuffizienz. Die Therapie der CSR erfolgt nach Optimierung der kardiologischen Therapie der Herzinsuffizienz und der ihr zu Grunde liegenden Ursachen idealerweise mit speziellen, nicht-invasiven Beatmungstherapie-Verfahren. Eine multizentrische und prospektiv-randomisierte Langzeit-Interventionsstudie, die aktuell durchgeführte Serve-HF-Studie, wird die Erkenntnisse über den Nutzen einer Therapie der CSR hinsichtlich des Überlebens der betroffenen Patienten vertiefen.

\section{Interessenkonflikte \\ $\nabla$}

Keine angegeben.

\section{Literatur}

1 American Academy of Sleep Medicine. ICSD-2 - International classification of sleep disorders, 2nd ed. Diagnostic and coding manual. Westchester, Illinois: American Academy of Sleep Medicine, 2005

2 Lévy P, Pépin JL, Arnaud C et al. Intermittend hypoxia and sleep-disordered breathing: current concepts and perspectives. Eur Respir J 2008; 32: $1082-1095$

3 Ulrich S, Fischler M, Speich R et al. Sleep-related breathing disorders in patients with pulmonary hypertension. Chest 2008; 133: 1375-1380

4 Eckert DJ, Jordan AS, Merchia P et al. Central sleep apnea - pathophysiology and treatment. Chest 2007; 131: 595-607

5 Naughton M, Benard D, Tam A et al. Role of hyperventilation in the pathogenesis of central sleep apneas in patients with congestive heart failure. Am Rev Respir Dis 1993; 148: 330 - 338

6 Bradley TD, Floras JS. Sleep apnea and heart failure: Part II: central sleep apnea. Circulation 2003; 107: 1822 -1826

7 Hall MJ, Xie A, Rutherford R et al. 1996. Cycle length of periodic breathing in patients with and without heart failure. Am J Respir Crit Care Med 1996; 154: 376- 381

8 Tkacova R, Niroumand M, Lorenzi-Filho G et al. Overnight shift from obstructive to central apneas in patients with heart failure: Role of $\mathrm{PCO}_{2}$ and circulatory delay. Circulation 2001; 103: 238-243

9 Steiner S, Schwartzkopff B, Strauer BE et al. Schlafapnoe: Risiko für die Herzinsuffizienz - Pathophysiologie, Diagnostik und Therapie. Dtsch Med Wochenschr 2005; 130: 468-472

10 Wang H, Parker JD, Newton GE et al. Influence of obstructive sleep apnea on mortality in patients with heart failure. J Am Coll Cardiol 2007; 49: $1625-1631$

$11 \mathrm{Xi}$ L, Smith CA, Saupe KW et al. Effects of rapid-eye-movement sleep on the apneic threshold in dogs. J Appl Physiol 1993; 75: 1129-1139

12 Sin DD, Fitzgerald F, Parker JD et al. Risk factors for central and obstructive sleep apnea in 450 men and women with congestive heart failure. Am J Crit Care Med 1999; 160: 1101 - 1106

13 Szollosi I, Thompson BR, Krum $\mathrm{H}$ et al. Impaired pulmonary diffusion capacity and hypoxia in heart failure correlates with central sleep apnea severity. Chest 2008; 134: 67-72

14 Garcia-Touchard A, Somers VK, Olson LJ et al. Central sleep apnea: implications for congestive heart failure. Chest 2008; 133: 1495-1504

15 Bitter M, Langer C, Vogt J et al. Schlafbezogene Atemstörungen - Charakteristika bei Vorhofflimmern und erhaltener linksventrikulärer Pumpfunktion. Dtsch Arztebl Int 2009; 106: 164 - 170

16 Gozal D, Kheirandish-Gozal L. Cardiovascular morbidity in obstructive sleep apnea. Am J Respir Crit Care Med 2008; 177: 369-375

17 Bundesärztekammer und Kassenärztliche Bundesvereinigung. Die stationäre Behandlung in Deutschland 2006. Dtsch Arztebl Int 2008; 105: B534

18 Levy D, Kenchaiah S, Larson MG et al. Long-term trends in the incidence of and survival with heart failure. N Engl J Med 2002; 347: 1397-1402

19 Oldenburg O, Lamp B, Faber $L$ et al. Sleep-disordered breathing in patients with symptomatic heart failure - a contemporary study of prevalence in and characteristics of 700 patients. Eur J Heart Fail 2006; 9: $251-257$

20 Schulz R, Blau A, Börgel J et al. Sleep apnoea in heart failure - results of a German survey. Eur Respir J 2007; 29: 1201 - 1205

21 Corrà U, Pistono $M$, Mezzani A et al. Sleep and exertional periodic breathing in chronic heart failure. Circulation 2006; 113: 44-50

22 Brack T, Thüer I, Clarenbach CF et al. Daytime Cheyne-Stokes respiration in ambulatory patients with severe congestive heart failure is associated with increased mortality. Chest 2007; 132: 1463-1471

23 Lanfranchi PA, Braghiroli A, Bosimini E et al. Prognostic value of nocturnal Cheyne-Stokes respiration in chronic heart failure. Circulation 1999; 99: 1435 - 1440

24 Javaheri S, Shukla $R$, Zeigler $H$ et al. Central sleep apnea, right ventricular dysfunction, and low diastolic blood pressure are predictors of mortality in systolic heart failure. J Am Coll Cardiol 2007; 49: 2028 2034

25 Duchna HW, Grote L, Andreas S et al. und Arbeitsgruppe Kreislauf und Schlaf der DGSM. State of the art article: Sleep-disordered breathing and cardio- and cerebrovascular diseases: update 2003 of clinical significance and future perspectives. Somnologie 2003; 7: 101-121

26 Hoppe UC, Böhm M, Dietz R et al. Vorstand der Deutschen Gesellschaft für Kardiologie - Herz- und Kreislaufforschung e.V. Leitlinien zur Therapie der chronischen Herzinsuffizienz. Z Kardiol 2005; 94: 488-509 
27 Dickstein K, Cohen-Solal A, Filippatos G et al. ESC guidelines for the diagnosis and treatment of acute and chronic heart failure 2008. Eur Heart J 2008; 29: 2388-2442

28 Teschler H, Döhring J, Wang Y et al. Adaptive pressure support servoventilation. Am J Respir Crit Care Med 2001; 164: 614-619

29 Schönhofer B, Kuhlen R, Neumann P et al. Nichtinvasive Beatmung als Therapie der akuten respiratorischen Insuffizienz - S3-Leitlinie herausgegeben von der Deutschen Gesellschaft für Pneumologie und Beatmungsmedizin. Pneumologie 2008; 62: 449-479

30 Abraham WT. Cardiac resynchronization therapy for the management of chronic heart failure. Am Heart Hosp J 2003; 1: 55-61

31 Sinha AM, Skobel EC, Breithardt OA et al. Cardiac resynchronization therapy improves central sleep apnea and Cheyne-Stokes respiration in patients with chronic heart failure. J Am Coll Cardiol 2004; 44: 68 71

32 Gabor YJ, Newman DA, Barnard-Roberts $V$ et al. Improvement in Cheyne-Stokes respiration following cardiac resynchronisation therapy. Eur Respir J 2005; 26: 95-100

33 Skobel E, Norra C, Sinha AM et al. Impact of sleep-related breathing disorders on health-related quality of life in patients with chronic heart failure. Eur J Heart Fail 2005; 7: 505-511

34 Bradley DJ, Bradley EA, Baughman KL et al. Cardiac resynchronization and death from progressive heart failure: a meta-analysis of randomized controlled trials. J Am Med Ass 2003; 289: 730-740

35 Duchna HW, Orth M, Karschuck P et al. Akuteffekte einer kardialen Resynchronisationstherapie auf die Atmung im Schlaf bei Patienten mit fortgeschrittener Herzinsuffizienz. Pneumologie 2008; 62: 189-195

36 Kara T, Novak M, Nykodym J et al. Short-term effects of cardiac resynchronisation therapy on sleep-disordered breathing in patients with systolic heart failure. Chest 2008; 134: 87-93

37 Bradley TD, Logan AG, Kimoff RJ et al. CANPAP Investigators. Continuous positive airway pressure for central sleep apnea and heart failure. $\mathrm{N}$ Engl J Med 2005; 353: 2025 - 2033

38 Arzt M, Floras JS, Logan AG et al. CANPAP-investigators. Suppression of central sleep apnea by continuous positive airway pressure and transplant-free survival in heart failure. Circulation 2007; 115: 3173-3180

39 Köhnlein T, Welte T, Tan LB et al. Assisted ventilation for heart failure patients with Cheyne-Stokes respiration. Eur Respir J 2002; 20: $934-$ 941

40 Morgenthaler TI, Gay PC, Gordon N et al. Adaptive servoventilation versus noninvasive positive pressure ventilation for central, mixed, and complex sleep apnea syndromes. Sleep 2007; 30: 468 - 475

41 Arzt M, Wensel R, Montalvan S et al. Effects of dynamic bilevel positive pressure support on central sleep apnea in men with heart failure. Chest 2008; 134: 61-66

42 Pepperell JC, Maskell NA, Jones DR et al. A randomized controlled trial of adaptive ventilation for Cheyne-Stokes breathing in heart failure. Am J Respir Crit Care Med 2003; 168: 1109-1114

43 Schädlich S, Königs I, Kalbitz F et al. Kardiale Leistungsfähigkeit bei Patienten mit Cheyne-Stokes-Atmung infolge Herzinsuffizienz während langfristiger nasaler Beatmungstherapie mittels adaptiver Servoventilation (AutoSet CS ${ }^{\circledR}$ ). Z Kardiol 2004; 93: 454-462

44 Noda A, Izawa $\mathrm{H}$, Asano $\mathrm{H}$ et al. Beneficial effect of bilevel positive airway pressure on left ventricular function in ambulatory patients with idiopathic dilated cardiomyopathy and central sleep apnea-hypopnea: a preliminary study. Chest 2007; 131: $1694-1701$

45 Arzt M, Bradley TD. Pulmonary perspective - Treatment of sleep apnea in heart failure. Am J Respir Crit Care Med 2006; 173: 1300-1308

\section{Information}

Liebe Kolleginnen und Kollegen,

die zweite Herbsttagung fand nach Gründung der Sektion Zellbiologie bereits in Borstel statt. Nachdem unser Herrenhaus renoviert ist, bietet Borstel nun erst recht einen idealen Ort zur Durchführung unseres Treffens. Wir möchten Sie sehr daher herzlich zum diesjährigen Herbsttreffen der Sektion Zellbiologie am 6. und 7. November nach Borstel einladen.

Die Einladung zur Tagung richtet sich zwar vor allem an Pneumologen und den pneumologischen Nachwuchs, ist aber interdisziplinär für alle Kliniker und Wissenschaftler mit Interesse an der pneumologischen Grundlagenforschung gedacht. Anders als im letzten Jahr haben wir uns dazu entschlossen, die Arbeiten wieder nur als Kurzvorträge präsentieren zu lassen, damit genug Zeit für die Darstellung und Diskussion der Projekte bleibt. Um die Diskussion zu stimulieren, werden wir jedem Abstract zwei Referenten zuweisen, die sich mit dem Abstract besonders auseinandersetzen und entsprechende Fragen vorbereiten. Diese Fragen sollen der Stimulation der Diskussion dienen, sie sollen keineswegs die Diskussion im Plenum ersetzen.

Die Herbsttagung lebt von der Beteiligung der Teilnehmerinnen und Teilnehmer. Wir bitten daher um Zusendung von Abstracts bis zum 30. September 2009.

Wie jedes Jahr wird der Freitagabend traditionell dem geselligen Beisammensein und dem Erfahrungsaustausch dienen. Hierzu dürfen wir Sie herzlich zum Abendessen einladen, das im Keller des Herrenhauses stattfinden wird.

Das endgültige Programm wird nach Eingang der Tagungsbeiträge im Oktober 2009 erstellt und auf der Webseite der Sektion Zellbiologie unter „Programm“ hinterlegt.

Nutzen Sie die Gelegenheit, mit anderen Arbeitsgruppen und Experten in Borstel ins Gespräch zu kommen. Wir freuen uns schon heute auf eine spannende zellbiologische Tagung im Herbst.

Mit den besten Grüßen,

Ihre

Prof. Dr. P. Zabel und PD Dr. H.-P. Hauber 